\title{
A NONLINEAR DIFFERENTIAL OPERATOR SERIES THAT COMMUTES WITH ANY FUNCTION*
}

\author{
PETER J. OLVER $\dagger$
}

\begin{abstract}
A natural differential operator series is one that commutes with every function. The only linear examples are the formal series operators $e^{\alpha z D}$ representing translations. This paper discusses a surprising natural nonlinear "normally ordered" differential operator series, arising from the Lagrange inversion formula. The operator provides a wide range of new higher-order derivative identities and identities among Bell polynomials. These identities specialize to a large variety of interesting identities among binomial coefficients and classical orthogonal polynomials, a number of which are new.
\end{abstract}

Key words. natural differential operator series, normal ordering, Lagrange inversion, Bell polynomial, orthogonal polynomial

AMS(MOS) subject classifications. 26A24, 05A15, 05A19

1. Introduction. An operator $\mathscr{D}$ is called natural if it commutes with arbitrary functions, i.e.,

$$
\Phi(\mathscr{D} u)=\mathscr{D} \Phi(u)
$$

for all scalar functions $\Phi$. In this paper we will take $u(t)$ to be a formal power series in the variable $t$, and $\mathscr{D}$ to be a formal series of differential operators. A simple example of a natural operator in this context is the exponential operator $e^{z D}$, where $D=d / d t$, which, by Taylor's theorem, coincides with the translation operator $e^{z D} u(t)=u(t+z)$. The proof that $e^{z D}$ is natural is then elementary:

$$
\Phi\left(e^{z D} u(t)\right)=\Phi(u(t+z))=e^{z D} \Phi(u(t)) .
$$

In fact, it is not hard to show that the translation operators $e^{\alpha z D}$ are essentially the only linear natural differential operator series. It is therefore rather surprising that there exist nonlinear natural differential operator series! The main result of this paper is that the series operator

$$
D^{-1}: e^{z D u}: D=1+\sum_{n=1}^{\infty} \frac{z^{n}}{n !} D^{n-1} \cdot u^{n} \cdot D
$$

is natural, i.e., for any analytic function $\Phi(u)$, and any formal power series $u(t)$,

$$
D^{-1}: e^{z D u}: D \Phi(u)=\Phi\left(D^{-1}: e^{z D u}: D u\right) .
$$

In (3) the colons mean that the operator is "normally ordered," meaning that all the multiplication terms appear after all the differentiations. This is reminiscent of the Wick ordering in quantum mechanics [4], although not quite the same. I do not know if the identity (4) has any bearing on this subject.

Two proofs of this identity will be discussed. The first is an application of the classical Lagrange inversion theorem [3], [7]. In fact, it will follow that the operator (3) formally represents the implicitly defined variable translation $x=t+z u(x)$, which explains its naturality. The second proof uses techniques from the Frobenius theory of partial differential equations, a method of independent interest, and appears in an

\footnotetext{
* Received by the editors January 3, 1990; accepted for publication (in revised form) February 21, 1991. This research was supported in part by National Science Foundation grant DMS 89-01600.

† School of Mathematics, University of Minnesota, Minneapolis, Minnesota 55455.
} 
appendix. By choosing different elementary functions $\Phi$ in the identity (4), we are led to a large class of interesting new identities involving higher-order derivatives of scalar functions. Moreover, specializing the resulting derivative identities to various elementary types of functions $u(x)$, leads to, among others, the Hagen-Rothe binomial coefficient identity [5], the Abel identity [3], and a number of interesting identities among classical orthogonal polynomials, including Hermite, Legendre, and Jacobi polynomials, that I have not been able to find in the literature. In another direction, using the standard connection between higher-order derivatives of compositions of functions and the Bell polynomials [3], [10], these derivative identities are easily shown to be equivalent to a large collection of apparently new identities for Bell polynomials.

This work arose from an ongoing investigation into the canonical forms for bi-Hamiltonian systems [9], and applications of these results to the precise integrability of canonical bi-Hamiltonian systems can be found there.

2. Differential operators and normal ordering. We will be concerned with formal power series whose coefficients are differential operators. These in turn can be applied to analytic functions or formal power series, leading in turn to further formal series.

Let $t$ be a scalar variable. We use $D$ to denote the derivative operator $d / d t$. Let

$$
F(z)=\sum_{n=0}^{\infty} c_{n} z^{n}
$$

be a formal power series in the scalar variable $z$. We can form the operator series

$$
F(z D)=\sum_{n=0}^{\infty} c_{n} z^{n} D^{n}
$$

which, when applied to any analytic function $f(t)$ results in a formal power series

$$
F(z D) f(t)=\sum_{n=0}^{\infty} c_{n} z^{n} f^{(n)}(t)
$$

in the derivatives of $f^{(n)}=D^{n} f=d^{n} f / d t^{n}$ of $f$. For example, by Taylor's theorem, the exponential operator

$$
e^{z D} f(t)=\sum_{n=0}^{\infty} \frac{1}{n !} z^{n} f^{(n)}(t)=f(t+z)
$$

coincides with the operator of translation in $z$. If

$$
f(t)=\sum_{i=0}^{\infty} f_{i} t^{i}
$$

is itself a formal power series, then (6) is a formal power series in both $z$ and $t$ whose coefficients depend on the coefficients $f_{i}$ of $f$. In particular, evaluating this identity at $t=0$ leads to the formal series

$$
\left.F(z D) f(t)\right|_{t=0}=\sum_{n=0}^{\infty} n ! c_{n} f_{n} z^{n} .
$$

Note that, under the natural identification of the coefficients of $f$ with the derivatives of $f$ at $t=0$, which is $f_{n}=(n !)^{-1} f^{(n)}(0)$, we recover the original equality (6). In fact, we can replace zero by any other value of $t$; hence we can use (8) to evaluate the series (6). This remark will be of use later on. 
We now wish to extend our range of operators to certain types of nonlinear operators. By "nonlinear" we mean that the operator itself depends on an analytic function or a formal power series $u(t)$, so that the operator will, in general, be a nonlinear function of $u$. However, it still acts linearly when applied to other power series. The most elementary operators associated with a formal power series (5) are the nonlinear operators

$$
F(z u D)=\sum_{n=0}^{\infty} c_{n} z^{n}(u D)^{n} \quad \text { and } \quad F(z D u)=\sum_{n=0}^{\infty} c_{n} z^{n}(D \cdot u)^{n}
$$

Note that since the operators of differentiation $D$ and multiplication by $u$ do not commute, these two operators are not the same; their commutator

$$
[D, u]=D \cdot u-u \cdot D=u^{\prime}
$$

is the operator of multiplication by $u^{\prime}=u^{(1)}$. For example,

$$
\begin{aligned}
e^{z u D} v= & \sum_{n=0}^{\infty} \frac{z^{n}}{n !}(u D)^{n} v \\
= & v+z u v^{\prime}+\frac{1}{2} z^{2}\left(u^{2} v^{\prime \prime}+u u^{\prime} v^{\prime}\right) \\
& +\frac{1}{6} z^{3}\left(u^{3} v^{\prime \prime \prime}+3 u^{2} u^{\prime} v^{\prime \prime}+u u^{\prime 2} v^{\prime}+u^{2} u^{\prime \prime} v^{\prime}\right)+\cdots, \\
e^{z D u} v= & \sum_{n=0}^{\infty} \frac{z^{n}}{n !}(D \cdot u)^{n} v \\
= & v+z\left(u v^{\prime}+u^{\prime} v\right)+\frac{1}{2} z^{2}\left(u^{2} v^{\prime \prime}+3 u u^{\prime} v^{\prime}+\left(u u^{\prime \prime}+u^{\prime 2}\right) v\right) \\
& +\frac{1}{6} z^{3}\left(u^{3} v^{\prime \prime \prime}+6 u^{2} u^{\prime} v^{\prime \prime}+\left(4 u^{2} u^{\prime \prime}+7 u u^{\prime 2}\right) v^{\prime}\right. \\
& \left.\quad+\left(u^{2} u^{\prime \prime \prime}+4 u u^{\prime} u^{\prime \prime}+u^{\prime 3}\right) v\right)+\cdots .
\end{aligned}
$$

A further type of operator is found by ordering the factors in the series in yet another way.

DEFINITION 1. Given a formal power series (5), the normally ordered operator series is defined to be

$$
: F(z D u):=\sum_{n=0}^{\infty} c_{n} z^{n} D^{n} \cdot u^{n}
$$

Thus the action of : $F(z D u)$ : on a function $f(t)$ is given by

$$
: F(z D u): f=\sum_{n=0}^{\infty} c_{n} z^{n} D^{n}\left\{u^{n} f\right\}
$$

The colons in the notation (11) are to distinguish this operator from the more standard operator series (9). For example

$$
\begin{aligned}
: e^{z D u}: v= & \sum_{n=0}^{\infty} \frac{1}{n !} z^{n} D^{n}\left(u^{n} v\right) \\
= & v+z\left(u v^{\prime}+u^{\prime} v\right)+\frac{1}{2} z^{2}\left(u^{2} v^{\prime \prime}+3 u u^{\prime} v^{\prime}+2\left(u u^{\prime \prime}+u^{\prime 2}\right) v\right) \\
& +\frac{1}{6} z^{3}\left(u^{3} v^{\prime \prime \prime}+9 u^{2} u^{\prime} v^{\prime \prime}+\left(9 u^{2} u^{\prime \prime}+18 u u^{\prime 2}\right) v^{\prime}\right. \\
& \left.+\left(3 u^{2} u^{\prime \prime \prime}+18 u u^{\prime} u^{\prime \prime}+6 u^{\prime 3}\right) v\right)+\cdots
\end{aligned}
$$


The colon notation is borrowed from quantum mechanics. Indeed, these operators remind us of the Wick ordering used in quantum field theory [4], in which all the creation operators appear to the left of all the annihilation operators. (Indeed, the commutation relations (10) are also reminiscent of the standard commutation relations, but only coincide when $u=t$.) However, this is not really the ordering adopted here, since in the harmonic oscillator, the creation and annihilation operators are certain combinations of derivative and multiplication operators.

3. Natural operators. Certain formal series differential operators play a distinguished role, in that they commute with functional evaluation. We make the following definition.

Definition 2. A series differential operator $\mathscr{D}$ is called natural if it commutes with all functions, i.e.,

$$
\Phi(\mathscr{D} u)=\mathscr{D} \Phi(u)
$$

for all scalar functions $\Phi$ and all formal series $u$.

A simple example is provided by the translation operator $e^{z D}$, as shown in the introduction. It is not hard to show that the following is essentially the only linear example.

Proposition 3. The operators $e^{\alpha z D}, \alpha$ a constant, are the only natural formal series linear differential operators of the form $\mathscr{D}=F(z D)$.

Proof. Let

$$
\mathscr{D}=F(z D)=\sum_{n=0}^{\infty} c_{n} z^{n} D^{n}
$$

First set $z=0$ in (12), which gives $\Phi\left(c_{0} u\right)=c_{0} \Phi(u)$. For this to hold for all $\Phi$, the leading term of $\mathscr{D}$ must be $c_{0}=1$. Now, assume by induction that we have shown that $c_{j}=\alpha^{j} / j$ !, for $j \leqq n-1$, where $\alpha=c_{1}$, and $n \geqq 2$. Let $\Phi(u)=u^{2}$. Then the coefficient of $z^{n}$ in (9) is

$$
2 c_{0} c_{n} u u^{(n)}+2 c_{1} c_{n-1} u^{\prime} u^{(n-1)}+\cdots=c_{n} D^{n}\left(u^{2}\right)
$$

This readily implies that $c_{n}=\alpha^{n} / n$ !, completing the induction. (Note that in fact we only needed to check (12) for quadratic functions $\Phi$ to prove this result.)

The main result of this paper is the following example of a nonlinear natural differential operator.

THEOREM 4. Let $u(t)$ be a formal power series and let $D=d / d t$. Then the series differential operator

$$
D^{-1}: e^{z D u}: D=1+\sum_{n=1}^{\infty} \frac{z^{n}}{n !} D^{n-1} \cdot u^{n} \cdot D
$$

is natural, i.e., for any analytic function $\Phi(u)$,

$$
D^{-1}: e^{z D u}: D \Phi(u)=\Phi\left(D^{-1}: e^{z D u}: D u\right) .
$$

Proof. This result follows as a direct consequence of the famous Lagrange inversion formula: cf. [3, p. 150] or [7, pp. 113, 114]. According to equation (5) of Melzak $[7$, p. 113], if $u(t)$ is any analytic function (or formal power series), and we define $x=\xi(z, t)$ implicitly by the formula

$$
x=t+z u(x),
$$


then, for any analytic function $f(t)$, we have the classical Lagrange inversion formula

$$
f(x)=f(t)+\sum_{n=1}^{\infty} \frac{z^{n}}{n !}\left\{D^{n-1} \cdot u(t)^{n} \cdot D\right\} f(t)=D^{-1}: e^{z D u}: D f(t) .
$$

Now set $f(t)=\Phi(u(t))$, so that (16) becomes

$$
\Phi(u(x))=D^{-1}: e^{z D u}: D \Phi(u(t)) .
$$

On the other hand, according to the formula at the bottom of page 144 of [7], for any analytic function $g(x)$, evaluated at (15),

$$
\left.\frac{\partial^{n}}{\partial z^{n}} g(\xi(z, t))\right|_{z=0}=D^{n-1}\left(u(t)^{n} D g(t)\right), \quad n \geqq 1 \text {. }
$$

Therefore, taking $g=u$, we find the expansion

$$
\begin{aligned}
u(x) & =\left.\sum_{n=0}^{\infty} \frac{z^{n}}{n !} \frac{\partial^{n}}{\partial z^{n}} u(\xi(z, t))\right|_{z=0} \\
& =u(t)+\sum_{n=0}^{\infty} \frac{z^{n}}{n !} D^{n}\left(u(t)^{n} D u(t)\right) \\
& =D^{-1}: e^{z D u}: D u(t) .
\end{aligned}
$$

Substituting this into (17) completes the proof of (13).

In view of the proof, then, it is no longer surprising that the operator series (13) is natural, since it corresponds to the variable translation (15) via Lagrange inversion. More generally, we can introduce the translation

$$
x=t+\Psi\left(z, u(x), u^{\prime}(x), \cdots, u^{(n)}(x)\right),
$$

which has a corresponding differential operator series, which will also clearly be natural. For example, the operators

$$
D^{-1}: e^{z D \Psi\left(u, u^{\prime}, \cdots\right)}: D,
$$

where $\Psi$ is any analytic function of $u$ and its derivatives, are also natural. An interesting problem that $I$ have not tried to investigate is whether there exist other classes of natural differential operators, although it seems reasonable to conjecture that only the operators associated with such translations are natural.

4. Derivative identities. Just as generating function identities leads to combinatorial identities, so any natural differential operator leads to a large class of derivative identities, obtained by considering different functions $\Phi$ in the basic condition (14). Here we present some of the more elementary derivative identities to be found as a consequence of the main theorem. We first compute the basic formula

$$
\begin{aligned}
\zeta(u)=D^{-1}: e^{z D u}: D u & =u+\sum_{n=1}^{\infty} \frac{z^{n}}{n !} D^{n-1}\left\{u^{n} u^{\prime}\right\} \\
& =\sum_{n=0}^{\infty} \frac{z^{n}}{(n+1) !} D^{n}\left(u^{n+1}\right) .
\end{aligned}
$$

More generally, we find that, for $\Phi(u)=u^{k}$,

$$
D^{-1}: e^{z D u}: D u^{k}=\sum_{n=0}^{\infty} \frac{k}{n+k} \frac{z^{n}}{n !} D^{n}\left(u^{n+k}\right) .
$$


As long as $k$ is not a negative integer, (20) is valid as it stands. It also remains correct when $k=-j$ is a negative integer, provided we interpret the term corresponding to $n=j$ in the summation according to the general "rule"

$$
\lim _{m \rightarrow 0} \frac{1}{m} D^{n}\left(u^{m}\right)=\lim _{m \rightarrow 0} D^{n-1}\left(u^{m-1} u^{\prime}\right)=D^{n}(\log u), \quad n \geqq 1 .
$$

Note that if $n=0$, the term $(k /(n+k)) D^{n}\left(u^{n+k}\right)=u^{k}$ is not a problem. Now, according to Theorem 4, the series (20) is the $k$ th power of the series (19). This implies certain unusual identities among higher-order derivatives of powers of $u$. For instance, taking the case $k=2$, the series identity

$$
\sum_{n=0}^{\infty} \frac{2}{n+2} \frac{z^{n}}{n !} D^{n}\left(u^{n+2}\right)=\left(\sum_{n=0}^{\infty} \frac{z^{n}}{(n+1) !} D^{n}\left(u^{n+1}\right)\right)^{2}
$$

implies the following derivative identities:

$$
D^{n}\left(u^{n+2}\right)=\sum_{i=0}^{n} \frac{n+2}{2(i+1)(n-i+1)}\left(\begin{array}{l}
n \\
i
\end{array}\right) D^{i}\left(u^{i+1}\right) \cdot D^{n-i}\left(u^{n-i+1}\right) .
$$

More generally, if we apply the identities corresponding to $\Phi(u)$ being $u^{k+l}, u^{k}$, and $u^{l}$, then the series identity

$$
\sum_{n=0}^{\infty} \frac{k+l}{n+k+l} \frac{z^{n}}{n !} D^{n}\left(u^{n+k+l}\right)=\left(\sum_{n=0}^{\infty} \frac{k}{n+k} \frac{z^{n}}{n !} D^{n}\left(u^{n+k}\right)\right)\left(\sum_{n=0}^{\infty} \frac{l}{n+l} \frac{z^{n}}{n !} D^{n}\left(u^{n+l}\right)\right)
$$

implies the additional derivative identities

$$
\begin{aligned}
& \frac{k+l}{n+k+l} D^{n}\left(u^{n+k+l}\right) \\
& \quad=\sum_{i=0}^{n} \frac{k l}{(i+k)(n-i+l)}\left(\begin{array}{c}
n \\
i
\end{array}\right) D^{i}\left(u^{i+k}\right) \cdot D^{n-i}\left(u^{n-i+l}\right) .
\end{aligned}
$$

These identities are valid for arbitrary (positive and negative) values of $k, l$, provided we use the rule (21) if either $n+k+l=0$, or any of the summation terms $i+k=0$ or $n-i+l=0$. For example, if we take $k=-1, l=1$, we find that the series

$$
\eta(u)=\frac{1}{u}-z \frac{u^{\prime}}{u}+\sum_{n=2}^{\infty} \frac{z^{n}}{(1-n) \cdot n !} D^{n}\left(u^{n-1}\right)
$$

is the series inverse for (19), i.e., $\eta(u)=1 / \zeta(u)$, and hence we have the series identity

$$
1=\left(\sum_{n=0}^{\infty} \frac{z^{n}}{(n+1) !} D^{n}\left(u^{n+1}\right)\right) \cdot\left(\frac{1}{u}-z \frac{u^{\prime}}{u}+\sum_{n=2}^{\infty} \frac{z^{n}}{(1-n) \cdot n !} D^{n}\left(u^{n-1}\right)\right) .
$$

Rearranging the terms of degree $n$ in $z$ in this formula results in the derivative identity

$$
D^{n}\left(u^{n+1}\right)=(n+1) u^{\prime} D^{n-1}\left(u^{n}\right)+\sum_{i=2}^{n} \frac{1}{i-1}\left(\begin{array}{c}
n+1 \\
i
\end{array}\right) u D^{i}\left(u^{i-1}\right) D^{n-i}\left(u^{n-i+1}\right),
$$

valid for $n \geqq 1$. The identities (22), (23) appear to be related to, but interestingly not the same as, some derivative identities appearing in Adams and Hippisley $[1, \S 7.37$, p. 160]. Many more examples can be deduced by choosing other types of elementary functions for $\Phi(u)$ in (14). 
5. Binomial and orthogonal polynomial identities. We now specialize the above derivative identities for particular functions $u(t)$, and find that they reduce to a wide range of identities among binomial coefficients and orthogonal polynomials. Some are known, but the orthogonal polynomial identities are apparently new. (However, I have not attempted a completely exhaustive search of the literature.)

1. First consider the case

$$
u(t)=t^{\alpha}, \quad \text { so } \frac{1}{n !} D^{n} u^{m}=\left(\begin{array}{c}
m \alpha \\
n
\end{array}\right) t^{m \alpha-n}
$$

Then (22) reduces to the identity

$$
\frac{k+l}{n+k+l}\left(\begin{array}{c}
(n+k+l) \alpha \\
n
\end{array}\right)=\sum_{i=0}^{n} \frac{k l}{(i+k)(n-i+l)}\left(\begin{array}{c}
(i+k) \alpha \\
i
\end{array}\right)\left(\begin{array}{c}
(n-i+l) \alpha \\
n-i
\end{array}\right) .
$$

This is equivalent to the Hagen-Rothe identity [5], [6, Eqn. 3.142], which generalizes the classical Vandermonde convolution identity for binomial coefficients,

$$
\left(\begin{array}{c}
r+s \\
n
\end{array}\right)=\sum_{i=0}^{n}\left(\begin{array}{c}
r \\
i
\end{array}\right)\left(\begin{array}{c}
s \\
n-i
\end{array}\right)
$$

which follows from (24) in the limit $\alpha \rightarrow 0, k \alpha \rightarrow r, l \alpha \rightarrow s$. In (24), we use the definition

$$
\left(\begin{array}{l}
\beta \\
n
\end{array}\right)=\frac{\beta(\beta-1) \cdots(\beta-n+1)}{n !}
$$

for the general binomial coefficients, so that

$$
\frac{1}{\beta}\left(\begin{array}{l}
\beta \\
n
\end{array}\right)=\frac{(\beta-1) \cdots(\beta-n+1)}{n !}
$$

is well defined even for $\beta=0$. As another example, the formula (23) in this case reduces to the identity

$$
\frac{1}{n+1}\left(\begin{array}{c}
(n+1) \alpha \\
n
\end{array}\right)=\alpha\left(\begin{array}{c}
n \alpha \\
n-1
\end{array}\right)+\sum_{i=2}^{n} \frac{1}{(i-1)(n-i+1)}\left(\begin{array}{c}
(i-1) \alpha \\
i
\end{array}\right)\left(\begin{array}{c}
(n-i+1) \alpha \\
n-i
\end{array}\right),
$$

which is similar to the Van der Corput identity; cf. [6, Eqn. 3.147].

2. Let

$$
u=e^{\alpha t}, \text { so } D^{n} u^{m}=m^{n} \alpha^{n} e^{\alpha t} .
$$

Then (22) reduces to the identity

$$
(n+k+l)^{n-1}=\sum_{i=0}^{n} \frac{k l}{k+l}\left(\begin{array}{l}
n \\
i
\end{array}\right)(i+k)^{i-1}(n-i+l)^{n-i-1} .
$$

If we set $k=-x / z, l=-n-(y / z)$, we deduce

$$
(x+y)^{n-1}=\sum_{i=0}^{n} \frac{x(y+n z)}{x+y+n z}\left(\begin{array}{c}
n \\
i
\end{array}\right)(x-i z)^{i-1}(y+i z)^{n-i-1},
$$

which is very similar to the Abel identity [3, p. 128],

$$
(x+y)^{n}=\sum_{i=0}^{n}\left(\begin{array}{l}
n \\
i
\end{array}\right) x(x-i z)^{i-1}(y+i z)^{n-i} .
$$


Indeed, they are essentially equivalent identities, since if we denote (27) by $A_{n}(x, y, z)$, and (26) by $B_{n-1}(x, y, z)$, then we easily verify the relation

$$
A_{n}(x, y, z)+n z A_{n-1}(x, y, z)=B_{n}(x, y, z) .
$$

Consequently, we can use Theorem 4 to give yet another proof of the Abel identity.

3. Let

$$
u=e^{-t^{2}}, \text { so } D^{n} u^{m}=(-1)^{n} m^{n / 2} H_{n}(\sqrt{m} t) e^{-m t^{2}},
$$

where $H_{n}$ denotes the usual Hermite polynomial [2, $\left.\S 10.13\right]$. In this case, $(22)$ reduces to the identity

$$
\begin{aligned}
& (n+k+l)^{(n / 2)-1} H_{n}(\sqrt{n+k+l} t) \\
& =\sum_{i=0}^{n} \frac{k l}{k+l}\left(\begin{array}{c}
n \\
i
\end{array}\right)(i+k)^{(i / 2)-1}(n-i+l)^{(n-i) / 2-1} H_{i}(\sqrt{i+k} t) H_{n-i}(\sqrt{n-i+l} t),
\end{aligned}
$$

which we can interpret as an Abel-type identity for Hermite polynomials. It is not the same as the usual addition formula, since the arguments of the Hermite polynomials appearing in the summation depend on the summation index $i$. If either $n+k+l=0$, $i+k=0$, or $n-i+l=0$, then we view the corresponding term in (28) according to the rule

$$
\lim _{m \rightarrow 0}(-1)^{n} m^{(n / 2)-1} H_{n}(\sqrt{m} t)= \begin{cases}-2 t, & n=1, \\ -2, & n=2, \\ 0, & n \geqq 3,\end{cases}
$$

stemming from the rule (21).

4. Let

$$
u=t^{\alpha} e^{-t}, \text { so } \frac{1}{n !} D^{n} u^{m}=t^{m \alpha-n} e^{-m t} L_{n}^{m \alpha-n}(m t)
$$

where $L_{n}^{\alpha}$ are the generalized Laguerre polynomials [2, § 10.12]. Again (22) reduces to an Abel-type identity

$$
\begin{aligned}
& \frac{k+l}{n+k+l} L_{n}^{(n+k+l) \alpha-n}((n+k+l) t) \\
& \quad=\sum_{i=0}^{n} \frac{k l}{(i+k)(n-i+l)} L_{i}^{(i+k) \alpha-i}((i+k) t) L_{n-i}^{(n-i+l) \alpha-n+i}((n-i+l) t)
\end{aligned}
$$

for Laguerre polynomials. As in the previous example, we make the convention

$$
\lim _{m \rightarrow 0} \frac{1}{m} L_{n}^{m \alpha-n}(m t)= \begin{cases}\alpha-t, & n=1, \\ {\left[(-1)^{n-1} / n\right] \alpha,} & n \geqq 2,\end{cases}
$$

stemming from the rule (21), for any exceptional terms in (29).

5. Finally, consider the case

$$
u=(1-t)^{\alpha}(1+t)^{\beta} .
$$


Then

$$
\frac{1}{n !} D^{n} u^{m}=(-2)^{n}(1-t)^{m \alpha-n}(1+t)^{m \beta-n} P_{n}^{(m \alpha-n, m \beta-n)}(t),
$$

where $P_{n}^{(\alpha, \beta)}$ are the Jacobi polynomials [2, $\left.\S 10.8\right]$. In this case (22) reduces to the Hagen-Rothe type formula

$$
\begin{aligned}
& \frac{k+l}{n+k}+l \\
& \quad P_{n}^{((n+k+l) \alpha-n,(n+k+l) \beta-n)}(t) \\
& \quad \sum_{i=0}^{n} \frac{k l}{(i+k)(n-i+l)} P_{i}^{((i+k) \alpha-i,(i+k) \beta-i)}(t) P_{n-i}^{((n-i+l) \alpha-n+i,(n-i+l) \beta-n+i)}(t),
\end{aligned}
$$

which again does not appear in the standard literature on Jacobi polynomials. Again, we need a rule

$$
\lim _{m \rightarrow 0} \frac{1}{m} P_{n}^{(m \alpha-n, m \beta-n)}(t)=\frac{\left[\alpha(t+1)^{n}-\beta(t-1)^{n}\right]}{\left[n(-2)^{n}\right]}, \quad n \geqq 1,
$$

for any exceptional terms in (30).

6. Bell polynomial identities. The Bell polynomials arise in the formula for the $n$th derivative of the composition of two functions [3], [10]. Specifically, we have

$$
\frac{d^{n}}{d t^{n}} f \circ g=\sum_{i=1}^{n}\left(f^{(i)} \circ g\right) \cdot B_{i}^{n}(g),
$$

where the $B_{i}^{n}$ are polynomials in the derivatives $g^{(k)}$ of $g$. Thus, the above derivative identities can be rewritten as identities involving Bell polynomials. Surprisingly, these identities have not appeared in the literature.

First, according to (31) (see [10, Ex. 22, p. 46]),

$$
\frac{d^{n}}{d t^{n}} u^{m}=\sum_{i=1}^{\min \{m, n\}} \frac{m !}{(m-i) !} u^{m-i} B_{i}^{n}(u) .
$$

Therefore, (19) can be rewritten as

$$
\zeta(u)=u+\sum_{n=1}^{\infty} \sum_{i=1}^{n} \frac{z^{n} u^{n+1-i}}{(n+1-i) !} B_{i}^{n}(u) .
$$

Furthermore, according to (14), (19), for $k$ a positive integer,

$$
\zeta(u)^{k}=u^{k}+\sum_{n=1}^{\infty} \sum_{i=1}^{n} \frac{k(n+k-1) !}{n !(n+k-i) !} z^{n} u^{n+k-i} B_{i}^{n}(u) .
$$

For example, if $k=2$, then

$$
\zeta(u)^{2}=u^{2}+\sum_{n=1}^{\infty} \sum_{i=1}^{n} \frac{2(n+1)}{(n+2-i) !} z^{n} u^{n+k-i} B_{i}^{n}(u) .
$$

If we compare this with the square of the series (33), we deduce that

$$
\frac{2(n+1)}{(n+2-i) !} B_{i}^{n}(u)=\frac{2}{(n+1-i) !} B_{i}^{n}(u)+\sum_{\substack{p+q=n \\ r+s=i}} \frac{1}{(p+1-r) !(q+1-s) !} B_{r}^{p}(u) B_{s}^{q}(u),
$$

which is equivalent to the identity

$$
2(i-1) B_{i}^{n}(u)=\sum_{p, r}\left(\begin{array}{c}
n+2-i \\
p+1-r
\end{array}\right) B_{r}^{p}(u) B_{i-r}^{n-p}(u) .
$$


By way of contrast, consider the Bell polynomial identity coming from squaring the standard exponential series

$$
e^{z u}=1+\sum_{n=1}^{\infty} \sum_{i=1}^{n} \frac{z^{i} t^{n}}{n !} B_{i}^{n}(u) .
$$

Equating $e^{2 z u}=\left(e^{z u}\right)^{2}$ and rearranging terms, we deduce

$$
2\left(2^{i-1}-1\right) B_{i}^{n}(u)=\sum_{p, r}\left(\begin{array}{l}
n \\
p
\end{array}\right) B_{r}^{p}(u) B_{i-r}^{n-p}(u) .
$$

which is quite similar to (23), but, except in very special cases, a different identity. More generally, the equation $e^{(a+b) z u}=e^{a z u} e^{b z u}$ leads to the further standard identities

$$
\left((a+b)^{i}-a^{i}-b^{i}\right) B_{i}^{n}(u)=\sum_{p, r}\left(\begin{array}{l}
n \\
p
\end{array}\right) a^{r} b^{i-r} B_{r}^{p}(u) B_{i-r}^{n-p}(u),
$$

valid for any $a, b$. On the other hand, the identity $\zeta(u)^{a+b}=\zeta(u)^{a} \zeta(u)^{b}$ leads to yet more complicated identities

$$
\begin{gathered}
{\left[\frac{(a+b)(n+a+b-1)}{a b}-\frac{(n+a-1)\left(\begin{array}{c}
n+a+b-i \\
b
\end{array}\right)}{b\left(\begin{array}{c}
n+a+b-i \\
b
\end{array}\right)}\right.} \\
\left.-\frac{(n+b-1)\left(\begin{array}{c}
n+a+b-i \\
a
\end{array}\right)}{a\left(\begin{array}{c}
n+a+b-i \\
a
\end{array}\right)}\right] B_{i}^{n}(u)
\end{gathered}
$$

More identities can be constructed by using different functions $\Phi(u)$ in the fundamental theorem. The number of different identities satisfied by the Bell polynomials is remarkable!

Appendix: Alternative proof of the main theorem. An alternative proof of Theorem 4 is based on the properties of certain first-order partial differential operators or vector fields; cf. [8]. As such, this proof may be adapted to give an alternative proof of the Lagrange inversion formula. The mathematical methods have not been used in the subject before, and thus are of some interest, possibly being of use in other problems. Let

$$
u=\sum_{n=0}^{\infty} u_{n} t^{n}
$$

be a formal power series. We will also work with the associated formal series

$$
v=\frac{1}{u}=\sum_{n=0}^{\infty} v_{n} t^{n}
$$

and

$$
w=\log \frac{u}{u_{0}}=\sum_{n=1}^{\infty} w_{n} t^{n} .
$$


We will regard the coefficients of $u$ and $v$, i.e., $u_{0}, u_{1}, u_{2}, \cdots$, and $v_{0}, v_{1}, v_{2}, \cdots$, as providing different local coordinates on the space of formal power series. They are connected by formulae of the form

$$
u_{n}=R_{n}\left(v_{0}, v_{1}, \cdots, v_{n}\right), \quad v_{n}=R_{n}\left(u_{0}, u_{1}, \cdots, u_{n}\right),
$$

where the rational functions $R_{n}$ can be explicitly expressed using determinants; cf. [10, Ex. 20, p. 45]. We can also use $u_{0}, w_{1}, w_{2}, \cdots$, as yet another set of coordinates, connected by formulae of the form

$$
u_{n}=u_{0} B_{n}\left(w_{1}, w_{2}, \cdots, w_{n}\right),
$$

where $B_{n}$ is a Bell polynomial coming from the relation $u=u_{0} e^{w}$. We now write out the basic series

$$
\Psi(u)=D^{-1}: e^{z D u}: D \Phi(u)=\sum_{n=0}^{\infty} z^{n} \Psi_{n},
$$

where, according to (13),

$$
\Psi_{n}=\left.\frac{1}{n !} D^{n} \Xi_{n}(u)\right|_{t=0}, \quad \Xi_{n}(u)=\int_{0}^{u} \tilde{u}^{n} \Phi^{\prime}(\tilde{u}) d \tilde{u} .
$$

Note that we are using the identification between series coefficients and derivatives given in (8) in this formula. In particular, the coefficient $\Psi_{n}$ depends on the first $n$ coefficients $u_{0}, u_{1}, \cdots, u_{n}$ of $u$. We can also re-express $\Psi_{n}$ in terms of the coefficients $v_{0}, v_{1}, \cdots, v_{n}$ of $v=1 / u$, or, alternatively, in terms of $u_{0}, w_{1}, w_{2}, \cdots, w_{n}$ using (A1). It will be very convenient to permit such changes of coordinates during the course of the proof.

The elementary first-order partial differential operators

$$
\mathbf{u}_{j}=\frac{\partial}{\partial u_{j}}, \quad \mathbf{v}_{j}=\frac{\partial}{\partial v_{j}}, \quad \mathbf{w}_{j}=\frac{\partial}{\partial w_{j}},
$$

will be regarded as vector fields acting on the functions of the coefficients of the formal power series $u$, and its associated power series $v=1 / u, w=\log \left(u / u_{0}\right)$. As such, they can all be re-expressed in any of our three coordinate systems. Note that

$$
\begin{aligned}
& \mathbf{v}_{j}(u)=-\mathbf{v}_{j}\left(\frac{1}{v}\right)=-\frac{t^{j}}{v^{2}}=-t^{j} u^{2}, \quad j=0,1,2, \cdots, \\
& \mathbf{w}_{j}(u)=\mathbf{w}_{j}\left(u_{0} e^{w}\right)=t^{j} u_{0} e^{w}=t^{j} u, \quad j=1,2,3, \cdots .
\end{aligned}
$$

Using these and similar formulae, it is not too difficult to verify the following change of variables formulae for these vector fields:

$$
\mathbf{v}_{j}=-\sum_{m, n=0}^{\infty} u_{m} u_{n} \frac{\partial}{\partial u_{m+n+j}}=-\sum_{n=0}^{\infty} u_{n} \frac{\partial}{\partial w_{n+j}}, \quad j=0,1,2, \cdots .
$$

(In the second summation, we use (A1) to re-express the $u$ 's in terms of the $w$ 's.) Also

$$
\mathbf{w}_{j}=\sum_{n=0}^{\infty} u_{n} \frac{\partial}{\partial u_{n+j}}=-\sum_{n=0}^{\infty} v_{n} \frac{\partial}{\partial v_{n+j}}, \quad j=1,2,3, \cdots
$$

Finally, we define the vector fields

$$
\mathbf{y}_{j}=\mathbf{w}_{j}+z \mathbf{v}_{j-1}, \quad j=1,2,3, \cdots,
$$

where $z$ is a scalar parameter. 
LeMma A1. The vector fields $\mathbf{y}_{j}$ all mutually commute:

$$
\left[\mathbf{y}_{j}, \mathbf{y}_{k}\right]=0 \text { for all } j, k=1,2,3, \cdots .
$$

The proof is simplest in the $v$ coordinates. We just re-express $\mathbf{w}_{j}$ using the second formula in (A7), and do a simple direct computation.

LemMA A2. Let $\Psi(u)=D^{-1}: e^{z D u}: D \Phi(u)$. Then

$$
\mathbf{y}_{j}[\Psi(u)]=0 \text { for all } j=1,2,3, \cdots .
$$

Proof. Note that since we are now working with formal power series, the vector fields $\mathbf{v}_{j}, \mathbf{w}_{j}$ commute with the derivative operator $D=d / d t$. Using (A3), (A4), we compute

$$
\begin{aligned}
\mathbf{v}_{j}[\Psi(u)] & =\left.\sum_{n=0}^{\infty} \frac{z^{n}}{n !} D^{n}\left\{\frac{\partial \Xi_{n}(u)}{\partial u} \mathbf{v}_{j}(u)\right\}\right|_{t=0} \\
& =-\left.\sum_{n=0}^{\infty} \frac{z^{n}}{n !} D^{n}\left\{t^{j} u^{n+2} \Phi^{\prime}(u)\right\}\right|_{t=0},
\end{aligned}
$$

whereas, using (A3), (A5), we find

$$
\begin{aligned}
\mathbf{w}_{j}[\Psi(u)] & =\left.\sum_{n=0}^{\infty} \frac{z^{n}}{n !} D^{n}\left\{\frac{\partial \Xi_{n}(u)}{\partial u} \mathbf{w}_{j}(u)\right\}\right|_{t=0} \\
& =\left.\sum_{n=0}^{\infty} \frac{z^{n}}{n !} D^{n}\left\{t^{j} u^{n+1} \Phi^{\prime}(u)\right\}\right|_{t=0} .
\end{aligned}
$$

Note that

$$
\left[D^{n}, t\right]=D^{n} \cdot t-t \cdot D^{n}=n D^{n-1}
$$

hence, upon evaluation at $t=0$,

$$
\left.D^{n} \cdot t\right|_{t=0}=\left.n D^{n-1}\right|_{t=0}
$$

Substituting this into the previous sum, we find (since $j \geqq 1$ )

$$
\begin{aligned}
\mathbf{w}_{j}[\Psi(u)] & =\left.\sum_{n=1}^{\infty} \frac{z^{n}}{(n-1) !} D^{n-1}\left\{t^{j-1} u^{n+1} \Phi^{\prime}(u)\right\}\right|_{t=0} \\
& =\left.\sum_{n=0}^{\infty} \frac{z^{n+1}}{n !} D^{n}\left\{t^{j-1} u^{n+2} \Phi^{\prime}(u)\right\}\right|_{t=0} .
\end{aligned}
$$

Comparing with the previous summation, we deduce that

$$
\mathbf{w}_{j}[\Psi(u)]=-z \mathbf{v}_{j-1}[\Psi(u)], \quad j=1,2,3, \cdots,
$$

which clearly implies the lemma.

If we write out the coefficient of $z^{n}$ in the previous formula (A11), we find that

$$
\mathbf{w}_{j}\left[\Psi_{n}(u)\right]=-\mathbf{v}_{j-1}\left[\Psi_{n-1}(u)\right], \quad j, n=1,2,3, \cdots .
$$

Since $\Psi_{n}$ only depends on $u_{0}, u_{1}, \cdots, u_{n}$, only the first $n$ of the equations in (A12) are nontrivial. We now regard (A12) as a system of first-order partial differential equations for the coefficients $\Psi_{n}$ of the series (A2). The commutativity Lemma A1 will imply that the system is in involution in the sense of Frobenius [8], and hence can be uniquely solved using suitable initial data. In fact, since the $w$ coordinates straighten out the vector fields $\mathbf{w}_{j}$, we can explicitly solve the system. 
Lemma A3. Let $\Psi_{n}, n=0,1,2, \cdots$, be functions depending on the coefficients $w_{j}$ of the formal series $w$ which satisfy

(i) $\quad \Psi_{n}\left(u_{0}, w_{1}, w_{2}, \cdots, w_{n}\right)$ depends only on the first $n$ coefficients of $w$.

(ii) $\quad \Psi_{n}\left(u_{0}, 0,0, \cdots, 0\right)=0$ for $n>0$.

$$
\frac{\partial \Psi_{n}}{\partial w_{j}}=\mathbf{v}_{j-1}\left[\Psi_{n-1}\right], \quad j=1, \cdots, n .
$$

Then $\Psi_{n}$ are uniquely determined by the function $\Psi_{0}\left(u_{0}\right)=\Phi\left(u_{0}\right)$.

Proof. According to Lemma A1, the integrability conditions for the elementary system of partial differential equations (iii) are satisfied. Therefore, the value of $\Psi_{n}$ is uniquely determined by its noncharacteristic Cauchy data prescribed by condition (ii). This completes the proof.

Now, to complete the proof of Theorem 4 , it suffices to notice that $\zeta(u)$, as defined by (A1), being a particular case of (A2), satisfies the three conditions of Lemma A3. But then the series $\Phi(\zeta(u))$ also satisfies them since, for example,

$$
\mathbf{y}_{j} \Phi(\zeta(u))=\Phi^{\prime}(\zeta(u)) \mathbf{y}_{j}(\zeta(u))=0 .
$$

Also, the leading-order term of $\Phi(\zeta(u))$ is $\Phi\left(u_{0}\right)$, which agrees with that of $\Psi(u)$ as given by (A2). According to the uniqueness result in Lemma A3, the series must agree, i.e., $\Phi(\zeta(u))=\Psi(u)$. This completes the proof of the main theorem.

Acknowledgments. I would like to express my gratitude to two anonymous referees, one for observing how the main theorem easily follows from Lagrange inversion, and the other for encouraging me to investigate the consequences of the new derivative identities (22), (23) which are discussed in $\S 5$.

\section{REFERENCES}

[1] E. P. AdAms AND R. L. Hippisley, Smithsonian Mathematical Formulae and Tables of Elliptic Functions, Smithsonian Miscellaneous Collections, Vol. 74, No. 1, Smithsonian Inst., Washington, D.C., 1922.

[2] H. Bateman, Higher Transcendental Functions, Vol. 2, A. Erdélyi, ed., McGraw-Hill, New York, 1953.

[3] L. Comtet, Advanced Combinatorics: The Art of Finite and Infinite Expansions, D. Reidel, Boston, MA, 1974.

[4] J. Glimm AND A. JAfFe, Quantum Physics, Second Ed., Springer-Verlag, New York, 1987.

[5] H. W. Gould, Some generalizations of Vandermonde's convolution, Amer. Math. Monthly, 63 (1956), pp. 84-91.

[6] - Combinatorial Identities, Morgantown, WV, 1972.

[7] Z. A. MelzaK, Companion to Concrete Mathematics, Wiley-Interscience, New York, 1973.

[8] P. J. Olver, Applications of Lie Groups to Differential Equations, Graduate Texts in Math. 107, Springer-Verlag, New York, 1986.

[9] — Canonical forms and integrability of biHamiltonian systems, Phys. Lett., 148A (1990), pp. 177-187.

[10] J. Riordan, An Introduction to Combinatorial Analysis, Princeton University Press, Princeton, NJ, 1980. 